\title{
A velocidade da marcha pode identificar idosos com medo de cair?
}

\author{
Can the gait speed identify elderly with fear of falling?
}

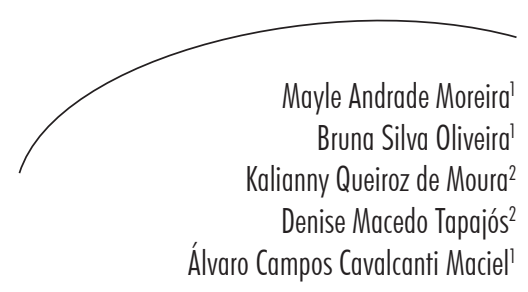

Resumo

Objetivo: Este estudo teve como propósito analisar a capacidade da velocidade da marcha em identificar idosos com medo de cair. Metodologia: Trata-se de estudo transversal analítico, realizado com amostra de 60 idosos da comunidade. Os dados foram obtidos por meio de questionário estruturado, análise da velocidade da marcha para percorrer a distância de 4,6 m e do medo de cair, avaliado pela escala de Autoeficácia de Quedas. A capacidade da velocidade da marcha em identificar idosos com medo de cair foi determinada por meio de regressão linear múltipla, com nível de significância $\mathrm{p}<0,05 \mathrm{e}$ IC 95\%. Resultados: Em relação à velocidade da marcha, a média foi de $0,71( \pm 0,21) \mathrm{m} / \mathrm{s}$, enquanto que para a escala de Autoeficácia de Quedas a média do escore foi de 24,85 $( \pm 6,68)$. Na análise de regressão linear múltipla, o escore total da escala de Autoeficácia de Quedas permaneceu significativamente associado $\left(\mathrm{R}^{2}=0,35\right)$ com a depressão autorrelatada, a limitação funcional para atividades básicas da vida diária e a velocidade da marcha. Conclusão: A velocidade da marcha, além da depressão autorrelatada e limitação funcional para atividades básicas da vida diária, têm capacidade de identificar idosos com medo de cair. Desta forma, a investigação das condições de saúde nessa população pode ser útil para detectar o medo de cair e identificar que cuidados e intervenções seriam prioritários para melhorar a funcionalidade e qualidade de vida desses idosos.

\section{Abstract}

Objective: This paper aimed to analyze the predictive ability of gait speed in identifying elderly with fear of falling. Methods: This is a cross-sectional analytical study conducted with a sample of 60 community elders. Data were collected through structured questionnaire, analysis of gait speed to walk a distance of $4.6 \mathrm{~m}$ and the fear of falling, which was assessed by the Falls Efficacy Scale. The predictive ability of the gait speed was determined by multiple linear regression, with significance level $\mathrm{p}<0.05$ and CI $95 \%$. Results: In relation to gait speed, the average was $0.71( \pm 0.21) \mathrm{m} / \mathrm{s}$, while for scale

Palavras-chave: Idoso. Marcha. Medo.
Key words: Elderly. Gait. Fear.

\footnotetext{
Programa de Pós-graduação em Fisioterapia. Universidade Federal do Rio Grande do Norte. Natal, RN, Brasil.

2 Curso de Fisioterapia. Centro de Ciências da Saúde. Universidade Federal do Rio Grande do Norte. Natal, RN, Brasil.
} 
of self-efficacy of falls the average score was 24.85 ( \pm 6.68$)$. In multiple linear regression analysis, the total score of the Falls Efficacy Scale remained significantly associated $\left(\mathrm{R}^{2}\right.$ $=0.35$ ) with self-reported depression, functional limitation for basic activities of daily living and gait speed. Conclusion: The gait speed beyond depression and self-reported functional limitation in basic activities of daily living, are able to identify elderly people with fear of falling. Thus, the investigation of the health in this population could be useful to detect the fear of falling, and identify what precautions are priority interventions to improve the functionality and quality of life of elderly people.

\section{INTRODUÇÃO}

O processo de envelhecimento é caracterizado por uma redução da capacidade física e funcional. Além disso, conforme o ser humano envelhece, sua massa muscular diminui, gerando instabilidade, perda da capacidade funcional e, sobretudo, aumento do risco de quedas, uma das principais causas de restrição em atividades da vida diária na terceira idade..$^{1-3}$

Quanto às consequências das quedas em idosos, estas não são somente físicas, mas também psicológicas, sociais e financeiras, como hospitalização, institucionalização ou reabilitação, aumento dos custos para a saúde pública, em termos de utilização de recursos e ocupação de leitos hospitalares. ${ }^{4,5}$ Segundo Ribeiro et al., ${ }^{6}$ entre as complicações mais citadas das quedas estão: as fraturas (24,3\%), o abandono de atividades (26,9\%), a modificação de hábitos $(23,1 \%)$, a imobilização $(19 \%)$ e principalmente o medo de cair $(88,5 \%)$.

Em relação às sequelas supracitadas, o medo de cair pode ser considerado uma das mais incapacitantes, ocasionando diminuição da mobilidade e aumento do desuso. Ou seja, existe um ciclo vicioso, no qual a inatividade pósqueda acentua a perda funcional, determinante na manutenção do equilíbrio postural, e por sua vez, essa perda de equilíbrio e medo de cair restringem a atividade cotidiana do idoso., ${ }^{4,7}$ Quanto às causas, embora não esteja claro, a literatura aponta para uma etiologia multifatorial do medo de cair, o qual está fortemente relacionado a fatores adversos que incluem qualidade de vida diminuída, redução da mobilidade, declínio de funcionalidade, aumento da fragilidade, depressão, fatores ambientais e institucionalização. ${ }^{7-9}$ Hauer et $a l .{ }^{5}$ destacam também a importância da função cognitiva, que inclui a atenção e concentração, para a manutenção do equilíbrio, pois o idoso pode não ter condições suficientes de cuidado para o controle postural.

Além disso, a velocidade da marcha vem sendo bastante discutida, pois sua redução está relacionada ao maior desequilíbrio e ao medo de cair. ${ }^{10}$ De forma geral, acredita-se que os idosos que caem durante a marcha podem ter maior risco de incapacidade em longo prazo, ${ }^{10}$ devido às consequências físicas, psicológicas e sociais que a queda traz ao idoso, que acabam culminando na restrição de atividades e perda da independência. ${ }^{4,5,11}$

Não obstante, com o envelhecimento, as pessoas tornam seus passos mais lentos e curtos, por ponderarem que melhoram a estabilidade e evitam as ameaças do desequilíbrio. No entanto, uma marcha com menor velocidade não traz tal benefício, mas sugere o maior risco de quedas. ${ }^{12}$

A tentativa de exercer controle sobre a marcha, com a redução do comprimento do passo, leva a instabilidade da cabeça e da pelve na direção vertical e ântero-posterior, afetando a estabilidade do ciclo da marcha. ${ }^{10,13}$ Diversas alterações são observadas, porém não está claro ainda se idosos com medo de cair reduzem a velocidade da marcha para evitar a queda ou se reduzem as atividades, como adaptação ao medo de cair, resultando em alteração na marcha. ${ }^{10}$

Neste sentido, a análise da velocidade da marcha vem sendo discutida como possível identificador de idosos com medo de cair, já que é um método de triagem de baixo custo, fácil 
reprodutibilidade, não necessita ser realizado em laboratório e possui valores, que podem ser analisados e comparados de maneira eficiente.

Diante do exposto, o objetivo do presente estudo foi analisar a capacidade da velocidade da marcha em identificar idosos com medo de cair.

\section{METODOLOGIA}

Este é um estudo transversal analítico, realizado na cidade de Natal - RN, no período de janeiro a abril de 2011, cujo desenho foi baseado nas recomendações do Reporting of Observational Studies in Epidemiology (STROBE), protocolo para estudos observacionais. ${ }^{14}$

A população foi composta por idosos residentes no bairro de Nova Descoberta, da zona urbana da cidade de Natal-RN. Os idosos foram recrutados de forma não-aleatória, compondo, entretanto, uma amostra representativa.

Os seguintes parâmetros estatísticos foram adotados para o cálculo do tamanho amostral: erros estatísticos máximos de 5,0\% para o tipo I e $20,0 \%$ para o erro de tipo II, com um poder de $80,0 \%$ para o estudo. Para um desvio de $5,0 \%$, para mais ou para menos, nas estimativas efetuadas e, considerando a população de referência de idosos com 65 anos ou mais e em condições de responder ao questionário, foi definida uma amostra de 60 idosos.

Os critérios de inclusão adotados foram: o idoso deveria apresentar 65 anos ou mais e ser capaz de caminhar de forma independente, mesmo com uso de dispositivos auxiliares; não apresentar deficiência visual ou auditiva, que impedisse a realização dos testes físicos e questionário; e não apresentar déficit cognitivo grave, que foi medido pelo Mini-Exame do Estado Mental (MEEM), o qual teve escore de 17 como ponto de corte, independente do grau de escolaridade. ${ }^{15}$ Uma vez selecionados, todos os 60 idosos completaram a avaliação.

A avaliação dos idosos foi realizada na própria residência do idoso, por entrevistadores previamente treinados, por meio de entrevista, utilizando um questionário estruturado, e análise da velocidade da marcha e força de preensão palmar. Antes de iniciar a entrevista, todos os participantes foram esclarecidos sobre os objetivos e procedimentos realizados, e todos assinaram o Termo de Consentimento Livre e Esclarecido, determinado pela Resolução $n^{\circ}$ 196/96 do Conselho Nacional de Saúde (CNS). O estudo foi aprovado pelo Comitê de Ética da Universidade Federal do Rio Grande do Norte, de acordo com o parecer de no 338/2010.

As variáveis independentes foram divididas nos seguintes grupos:

- Variáveis sociodemográficas: idade, sexo e escolaridade.

- Variáveis de saúde física: doenças crônicas autorrelatadas $^{16}$ (doença do coração, hipertensão, AVC, diabetes mellitus, artrite ou reumatismo e depressão), autopercepção da saúde, massa corporal, altura, força de preensão palmar, velocidade da marcha e número de quedas no último ano.

- Variáveis de capacidade funcional: escore de independência para atividades instrumentais da vida diária (AIVD), em que foi avaliada a total independência do idoso no que se refere à realização das AIVDs, como proposto pela escala desenvolvida por Lawton \& Brody $;{ }^{17}$ e o escore de independência para atividades básicas da vida diária (ABVD), em que se utilizou o Índice de Katz ${ }^{18}$ para avaliar a total independência em relação às ABVDs.

- Variável neuropsíquica: escore do MiniExame do Estado Mental (MEEM).${ }^{14}$ Foi utilizado o escore de valor 17 como ponto de corte, independentemente do grau de escolaridade, ${ }^{15} \mathrm{com}$ o objetivo de evitar, em decorrência de um ponto de corte muito alto, perder muitos idosos, além de excluir idosos sem condições de compreender os comandos para os testes físicos, caso o ponto de corte fosse muito baixo. 
A variável dependente foi a escala de Autoeficácia de Quedas, ${ }^{19}$ que é referente à Falls Efficacy Scale - International, FES-I. ${ }^{20}$ Este instrumento foi desenvolvido por Tinetti et al. ${ }^{20}$ para medir o medo de cair e é baseado na definição operacional de medo como percepção de diminuta autoconfiança para evitar quedas durante tarefas essenciais, potencialmente não lesivas. ${ }^{21}$ A FES-I foi validada no Brasil (FES-IBRASIL) $)^{19}$ e é um instrumento promissor no que diz respeito à avaliação do medo de cair tanto em pesquisas, quanto na prática clínica. ${ }^{19}$ Esta escala apresenta questões sobre a preocupação com a possibilidade de cair ao realizar 16 atividades, com respectivos escores de um a quatro, podendo o escore total variar de 16 (ausência de preocupação) a 64 (preocupação extrema). ${ }^{19}$

Dentre os instrumentos utilizados, para a medida da massa corporal foi utilizada uma balança Felizona, série 3.134, com divisões de 100g; para a tomada da altura, fitas métricas de fiber glass com divisões de $1 \mathrm{~mm}$ e a força de preensão palmar foi determinada por um dinamômetro manual hidráulico Jamar ${ }^{\circledR}$, modelo J00105.

Para a mensuração da força de preensão palmar, os idosos foram posicionados sentados, com o braço dominante aduzido paralelo ao tronco, cotovelo fletido a $90^{\circ}$ graus e antebraço e punho em posição neutra. Foram realizadas três medições, com intervalo de 30 segundos entre elas, e registrada a média aritmética dos valores medidos.

A análise da velocidade da marcha foi realizada com marcação do tempo, em milésimos de segundos, a partir do deslocamento do idoso em um percurso de 4,6 metros, em linha reta, baseando-se na medida utilizada como um dos itens para a composição do fenótipo de fragilidade. $^{8}$

Os dados foram armazenados e analisados pelo programa estatístico SPSS (Statistical Package for the Social Sciences), versão 17.0. A estatística descritiva foi realizada usando-se medidas de distribuição: médias e desvios-padrão para variáveis quantitativas e frequências absolutas e relativas para variáveis categóricas. Em seguida, foi realizada análise, por meio do teste de correlação de Pearson, entre os resultados da avaliação da FES-I-BRASIL e as variáveis independentes quantitativas. $\mathrm{Na}$ análise das médias dos escores da FES-I-BRASIL em relação às variáveis categóricas, foi utilizado o teste $t$ Student para amostras independentes. A terceira etapa da análise estatística consistiu na construção de modelos de regressão linear múltipla, com o objetivo de identificar os fatores que podem predizer as relações entre as variáveis independentes e a FES-I-BRASIL. O critério de saída para todas as variáveis introduzidas no modelo foi de $\mathrm{p}<0,10$. Em toda análise estatística, foi considerado um intervalo de confiança (IC) de $95 \%$ e um $\mathrm{p} \leq 0,05$.

\section{RESULTADOS}

A amostra foi composta por 60 idosos com média de idade de 77,1 anos $( \pm 5,6)$, sendo 27 $(45 \%)$ homens e $33(55 \%)$ mulheres. As demais características da amostra quanto às variáveis independentes estão descritas na tabela 1. Em relação à velocidade da marcha, a média foi de $0,71( \pm 0,21) \mathrm{m} / \mathrm{s}$, enquanto que para a FES-IBRASIL, a média do escore foi de $24,85( \pm 6,68)$. 
Tabela 1 - Características da amostra quanto às variáveis independentes. Natal, RN, 2011.

\begin{tabular}{cc}
\hline VARIÁVEIS & TOTAL \\
\hline Escolaridade (anos) & $5,2( \pm 3,7)$ \\
Cardiopatia (\%) & 20,0 \\
Hipertensão (\%) & 55,0 \\
AVC (\%) & 1,1 \\
Diabetes mellitus (\%) & 18,3 \\
Reumatismo (\%) & 44,8 \\
Depressão (\%) & 21,6 \\
Percepção da saúde (\%) & \\
Boa & 40,5 \\
Ruim & 55,4 \\
Escore de independência para AIVD & $9,1( \pm 2,8)$ \\
Escore de independência para ABVD & $6,2( \pm 3,2)$ \\
Massa corporal (kg) & $62,4( \pm 13,5)$ \\
Altura (cm) & $155,9( \pm 9,8)$ \\
IMC (kg/m $\left.{ }^{2}\right)$ & $25,7( \pm 4,19)$ \\
Força de preensão palmar (kgf) & $22,1( \pm 7,4)$ \\
Ocorrência de quedas no último ano (\%) & 51,7 \\
Escore do MEEM & $23,1( \pm 3,5)$ \\
\hline \hline
\end{tabular}

A tabela 2 apresenta as correlações entre as variáveis independentes quantitativas com a FES-I-BRASIL. Neste sentido, encontraram-se correlações estatísticas significativas na escolaridade, no MEEM, nas ABVD, nas AIVD, na força de preensão palmar e velocidade da marcha.

Tabela 2 - Correlações entre as variáveis independentes quantitativas com a FES-I-BRASIL. Natal, RN, 2011.

\begin{tabular}{lc}
\hline \multicolumn{1}{c}{ VARIÁVEIS } & FES-I-BRASIL \\
\hline Idade & $\mathrm{r}=0,24 ; \mathrm{p}=0,298$ \\
Escolaridade & $\mathrm{r}=-0,26 ; \mathbf{p}=\mathbf{0}, \mathbf{0 4}$ \\
MEEM & $\mathrm{r}=-0,45 ; \mathbf{p}<\mathbf{0 , 0 0 1}$ \\
$\mathrm{N}^{\text {o de quedas }}$ & $\mathrm{r}=0,23 ; \mathrm{p}=0,07$ \\
ABVD & $\mathrm{r}=0,53 ; \mathbf{p}=\mathbf{0 , 0 1}$ \\
AIVD & $\mathrm{r}=0,58 ; \mathbf{p}<\mathbf{0 , 0 0 1}$ \\
Altura & $\mathrm{r}=-0,21 ; \mathbf{p}=0,09$ \\
Massa corporal & $\mathrm{r}=-0,35 ; \mathbf{p}=0,21$ \\
Força de preensão palmar & $\mathrm{r}=-0,36 ; \mathbf{p}=\mathbf{0 , 0 0 5}$ \\
Velocidade da marcha & $\mathrm{r}=-0,36 ; \mathbf{p}=\mathbf{0 , 0 0 5}$ \\
\hline
\end{tabular}


Na tabela 3, são apresentadas as diferenças nas médias da FES-I-BRASIL em relação às variáveis independentes categóricas. Observouse diferença significativa apenas na variável depressão autorrelatada, onde os idosos com a presença deste quadro tiveram média mais alta em relação aos que não tinham a doença.

Tabela 3 - Análise das médias dos escores da FES-I-BRASIL em relação às variáveis independentes categóricas. Natal, RN, 2011.

\begin{tabular}{|c|c|c|}
\hline VARIÁVEIS & FES-I-BRASIL & $\mathrm{p}$ \\
\hline \multicolumn{3}{|c|}{ Doença do coração } \\
\hline $\operatorname{Sim}$ & $24,08( \pm 7,37)$ & 0,66 \\
\hline Não & $25,04( \pm 6,56)$ & \\
\hline \multicolumn{3}{|l|}{ HAS } \\
\hline $\operatorname{Sim}$ & $24,93( \pm 6,86)$ & 0,91 \\
\hline Não & $24,74( \pm 6,57)$ & \\
\hline \multicolumn{3}{|l|}{ AVC } \\
\hline $\operatorname{Sim}$ & $26,66( \pm 10,96)$ & 0,63 \\
\hline Não & $24,75(6,52)$ & \\
\hline \multicolumn{3}{|l|}{ Diabetes mellitus } \\
\hline Sim & $25,72( \pm 8,10)$ & 0,63 \\
\hline Não & $24,65(6,40)$ & \\
\hline \multicolumn{3}{|l|}{ Reumatismo } \\
\hline Sim & $25,58( \pm 7,17)$ & 0,49 \\
\hline Não & $24,36( \pm 6,39)$ & \\
\hline \multicolumn{3}{|l|}{ Depressão } \\
\hline Sim & $29,01( \pm 8,49)$ & 0,03 \\
\hline Não & $24,02( \pm 6,02)$ & \\
\hline \multicolumn{3}{|c|}{ Percepção de saúde } \\
\hline Boa & $23,36( \pm 5,74)$ & 0,20 \\
\hline Ruim & $25,71( \pm 6,87)$ & \\
\hline \multicolumn{3}{|c|}{ Ocorrência de queda no último ano } \\
\hline $\operatorname{Sim}$ & $24,41( \pm 6,43)$ & 0,61 \\
\hline Não & $25,31( \pm 7,02)$ & \\
\hline
\end{tabular}

Em seguida, na análise de regressão linear múltipla, o escore total da FES-I-BRASIL permaneceu significativamente associado
$\left(\mathrm{R}_{\text {ajustado }}^{2}=0,35, \beta=14,65\right)$ com a depressão autorrelatada, a velocidade da marcha e o escore de ABVD, de acordo com a tabela 4.

Tabela 4 - Resultado da análise de regressão linear múltipla com as variáveis preditivas do escore da FES-I-BRASIL. Natal, RN, 2011.

\begin{tabular}{cccc}
\hline VARIÁVEIS & $\boldsymbol{\beta}$ & ERRO-PADRÃO & $\mathbf{p}$ \\
\hline Depressão & $-4,84$ & 1,93 & 0,01 \\
Velocidade de marcha & $-12,06$ & 3,34 & 0,001 \\
Limitação para ABVD & 4,42 & 1,25 & 0,001 \\
\hline
\end{tabular}




\section{DISCUSSÃO}

A análise da velocidade da marcha em idosos é considerada importante indicador do nível de saúde. Esta medida é um instrumento eficiente para prever expectativa de vida, surgimento de incapacidade física, demência, quedas, além da necessidade de hospitalização, justamente por ser uma expressão da interação de diferentes órgãos e estruturas do corpo. ${ }^{22}$

No presente estudo, o valor encontrado da média da velocidade da marcha $(0,71 \mathrm{~m} / \mathrm{s})$ encontra-se abaixo do valor padrão $(0,8 \mathrm{~m} / \mathrm{s})$ estabelecido para uma expectativa de vida média. $^{23}$ Possivelmente, a elevada idade da amostra reduziu os valores encontrados, uma vez que é esperada, ao longo dos anos, uma redução de alguns parâmetros envolvidos na marcha, como comprimento do passo e velocidade. $^{10}$ Velocidades superiores à média predizem expectativa de vida além do mediano (acima de 1,2 m/s indica uma expectativa de vida excepcional) e velocidade da marcha menor que $0,5 \mathrm{~m} / \mathrm{s}$ prediz pior estado de saúde em idosos. ${ }^{23}$

Em relação à FES-I-BRASIL, a média do escore neste estudo $(24,85)$ se aproximou do valor encontrado no estudo de validação da escala no Brasil $(23,55) .{ }^{19}$ Outras pesquisas realizadas em Belo Horizonte e São Paulo, cujas amostras foram similares a do presente estudo, também apresentaram resultados aproximados, 24,01 e 26,5 , respectivamente. ${ }^{78}$ Já na pesquisa realizada com 25 idosos ativos em Uberlândia, a média do escore da FES-I-BRASIL alcançou valor maior $(31,0) \cdot{ }^{24}$ De uma forma geral, escores superiores a 23 pontos têm associação com a queda esporádica, ao passo que uma classificação superior a 30 tem associação com queda recorrente. ${ }^{19}$

$\mathrm{Na}$ análise de correlação observouse significância da FES-I-BRASIL com a escolaridade e a função cognitiva. Para Custódio et al., ${ }^{25}$ a baixa escolaridade e o envelhecimento associam-se à diminuição da capacidade cognitiva; portanto, um maior nível de escolaridade ocasiona maior ativação sináptica, com melhora na percepção, memória e raciocínio, o que retarda o aparecimento de demência. Da mesma forma, Hauer et al. ${ }^{5}$ afirmam que idosos com maior déficit cognitivo podem não ter condições de alocar recursos cognitivos relacionados à atenção suficientes para o controle postural, bem como podem ter dificuldades de realizar atividades como caminhar ou manter o controle postural, que possivelmente acabam gerando o medo de cair.

No presente estudo, observou-se também correlação significativa da FES-I-BRASIL com a força de preensão palmar. Este resultado corrobora o estudo de Soares et al., ${ }^{26}$ o qual observou que a força de preensão palmar, no grupo de caidores, foi significativamente menor do que no grupo de não-caidores; e que o escore da FES-I foi significativamente maior no grupo de caidores. ${ }^{26}$ Não obstante, o envelhecimento é caracterizado por um declínio na performance motora e pela diminuição gradual do movimento, sendo a fraqueza muscular um grande contribuinte para o declínio da funcionalidade do idoso. ${ }^{4}$ Uma diminuição da força muscular afeta a capacidade laboral, a atividade motora e a adaptabilidade ao ambiente, contribuindo para que ocorram instabilidades. ${ }^{4}$ Ou seja, o declínio na força muscular torna o idoso mais propenso a desfechos adversos de saúde como quedas, medo de cair, dependência de terceiros e incapacidade funcional. ${ }^{4,26}$

$\mathrm{Na}$ análise de regressão, o escore total da FES-I-BRASIL permaneceu significativamente associado com a limitação para as ABVD, depressão autorrelatada e a velocidade da marcha. Em relação à associação com as atividades básicas da vida diária (ABVD), que é um componente da avaliação da capacidade funcional associado às atividades instrumentais da vida diária (AIVD), tal achado foi compatível com os encontrados na literatura, como o estudo de Silva et al., ${ }^{12}$ o qual encontrou correlação significativa entre os escores da FES-I e a escala de Lowton $(r=0,565 ; \mathrm{p}=0,001){ }^{.}$

Já a pesquisa de Deshpande et al., ${ }^{27}$ realizada com 1.067 idosos, 673 participantes relataram medo de cair e, destes, cerca de 25\% dos participantes não relataram qualquer restrição de atividade, 59,6\% relataram moderada restrição 
de atividade e 14,9\% relataram restrição severa de atividade. ${ }^{27}$ De forma adicional, em outro estudo de Deshpande et al., quase 50\% dos participantes relataram medo de cair e, destes, 65\% relataram alguma restrição de atividade.

Nesse sentido, o prejuízo da capacidade funcional parece ter papel preponderante na interação multicausal de quedas. Os idosos com baixa autoconfiança em realizar atividades do dia a dia estão potencialmente sujeitos ao isolamento social e à aquisição de estilos de vida sedentários ao longo do tempo. Isso pode explicar o aumento no risco de quedas, bem como o medo de que elas ocorram, o que gera um ciclo.

No tocante à depressão autorrelatada, atualmente, os estudos têm definido o medo de cair como baixa autoeficácia ou baixa confiança em evitar queda. ${ }^{7}$ Pessoas com baixa autoconfiança tendem a focar muito mais suas limitações, enfatizando as deficiências. Acredita-se, portanto, que pessoas que resistem às situações ameaçadoras, fugindo delas, talvez impeçam o desenvolvimento de habilidades capazes de superar tais limitações, resultando em uma base para construção do medo. ${ }^{7}$

Dessa forma, o medo de cair é importante porque tem impacto negativo na vida do idoso. Essa perda de autoconfiança está associada muitas vezes com outros fatores psicológicos, como ansiedade e depressão, restrição de atividade e, finalmente, a perda de independência. ${ }^{11}$ Afirmase que todas as pessoas envolvidas com idosos, inclusive profissionais, devem motivar os mesmos mediante atividades sócio-interativas, no sentido de que estes possam se tornar ativos, diminuindo os fatores que levam à inatividade e depressão. ${ }^{4}$

Por fim, na análise da regressão, observouse que a velocidade da marcha permaneceu significativamente associada com a FES-IBRASIL. Sabe-se que a velocidade da marcha lenta, com menor comprimento do passo, maior base de suporte e maior tempo de duplo apoio podem estar associados com um medo preexistente de queda. Em concordância, o estudo de Chamberling et al. ${ }^{28}$ observou velocidade da marcha mais lenta no grupo que apresentava maior medo de cair, quando comparado ao grupo sem medo. Portanto, tal pesquisa apoia a hipótese de que existe relação entre o medo de cair e as alterações dos parâmetros espaciais e temporais da marcha em pessoas idosas. ${ }^{28}$

Lopes et al. ${ }^{7}$ destacam que os idosos com medo de cair utilizam estratégias para manter o equilíbrio durante a marcha, sendo mais cautelosos, abreviando o tempo de permanência na fase de oscilação, fase de maior instabilidade, além da diminuição do impulso, extensão de joelhos, diminuição do comprimento e altura do passo e, consequentemente, redução da velocidade, estratégias empregadas para diminuir o risco de quedas. ${ }^{7}$ Em situações de ameaça ou instabilidade, é natural que o idoso busque se proteger, reduzindo a velocidade da marcha, no entanto a velocidade normal é a que produz maior estabilidade. ${ }^{13}$

A relação complexa entre o medo de cair e a velocidade da marcha não é totalmente compreendida e, em geral, não foi amplamente investigada. Rochat et al. $^{29}$ mostram que a velocidade da marcha reduzida é associada com níveis elevados do medo de cair e sugerem que o medo, por si só, possa causar a restrição de atividade e ter efeito direto na marcha, independentemente do desempenho físico. $\mathrm{O}$ medo de cair pode resultar em recrutamento simultâneo dos músculos agonistas e antagonistas, resultando em uma espécie de rigidez postural, marcha anormal, estratégias posturais inadequadas, insegurança, dependência de dispositivos que garantam estabilidade (como órteses) e aumento do risco de quedas.?

Existe, porém, a hipótese de que a relação entre o desempenho da marcha e o medo de cair poderia ser recíproca, em vez de unidirecional. ${ }^{29}$ Não está claro ainda se idosos com medo de cair reduzem a velocidade da marcha para evitar a queda ou se reduzem as atividades, como adaptação ao medo de cair, resultando em alteração na marcha. ${ }^{10} \mathrm{O}$ que se sabe é que as medidas dos parâmetros da marcha, como a velocidade da marcha, ao longo de várias sessões, são indicadores fiéis da mobilidade no idoso $^{30}$ e têm capacidade preditiva em relação ao 
medo de cair, além de serem fortes preditores de eventos adversos, como futuras quedas, declínio funcional e até mesmo a mortalidade. ${ }^{29}$

Algumas possíveis limitações desta pesquisa devem ser consideradas, como o desenho transversal do estudo, que impossibilita estabelecer uma relação de causalidade, que seria possível através de um estudo longitudinal; o pequeno tamanho amostral $(\mathrm{n}=60)$ e a seleção da amostra por conveniência, e não de forma randomizada, fatores que podem limitar a validade externa dos resultados.

Apesar disso, a relevância de trabalhos como este deve ser mencionada, pois partindo do pressuposto de que idosos estão propensos a desenvolver o medo de cair e sofrer maior declínio funcional, os dados obtidos a partir desse tipo de pesquisa permitem traçar um perfil

\section{REFERÊNCIAS}

1. Gonçalves LHT, Silva AH, Mazo GZ, Benedetti TRB, Santos SMA, Marques S, et al. O idoso institucionalizado: avaliação da capacidade funcional e aptidão física. Cad. Saúde Pública 2010;26(9):1738-46.

2. Gazzola JM, Perracini MR, Ganança MM, Ganança FF. Functional balance associated factors in the elderly with chronic vestibular disorder. Braz J Otorhinolaryngol 2006;72(5):683-90.

3. Visvanathan R, Chapman I. Preventing sarcopaenia in older people. Maturitas 2010;66(4):383-8.

4. Menezes RL, Bachion MM. Estudo da presença de fatores de riscos intrínsecos para quedas, em idosos institucionalizados. Cienc Saude Coletiva 2008;13(4):1209-18.

5. Hauer K, Marburger C, Oster P. Motor performance deteriorates with simultaneously performed cognitive tasks in geriatric patients. Arch Phys Med Rehabil 2002;83(2):217-23.

6. Ribeiro AP, Souza ER, Atie S, Souza AC, Schilithz AO. Influência das quedas na qualidade de vida de idosos. Cienc Saude Coletiva 2008;13(4):1265-73.

7. Lopes KT, Costa DF, Santos LF, Castro DP, Bastone AC. Prevalência do medo de cair em uma população de idosos da comunidade e sua correlação com mobilidade, equilíbrio dinâmico, risco e histórico de quedas. Rev Bras Fisioter 2009;13(3):223-9. das condições gerais de saúde, e funcionalidade dessas pessoas, que pode ser essencial para auxiliar em prevenções de quedas e retardar agravos à saúde.

\section{CONCLUSÃO}

Observou-se, portanto, que uma forma prática, objetiva, quantificável e simples de identificar a presença do medo de cair pode ser a medida da velocidade da marcha, já que é uma avaliação de baixo custo e pode ser utilizada durante os atendimentos, inclusive para acompanhamento da progressão dos pacientes. Desta forma, a investigação das condições de saúde desses idosos pode ser útil para detectar o medo de cair e identificar que cuidados e intervenções seriam prioritários para melhorar a funcionalidade e qualidade de vida destes idosos.

8. Silva SLA, Vieira RA, Arantes P, Dias RC. Avaliação de fragilidade, funcionalidade e medo de cair em idosos atendidos em um serviço ambulatorial de geriatria e gerontologia. Fisioter Pesqui 2009;16(2):120-5.

9. Deshpande N, Metter EJ, Bandinelli S, Lauretani F, Windham BG, Ferrucci L. Psychological, physical, and sensory correlates of fear of falling and consequent activity restriction in the elderly: the In:CHIANTI study. Am J Phys Med Rehabil 2008;87(5):354-62.

10. Kirkwood RN, Moreira BS, Vallone MLDC, Mingoti AS, Dias RC, Sampaio RF. Step length appears to be astrong discriminant gait parameter for elderly females highly concerned about falls: a cross-sectional observational study. Physiotherapy 2011;97(2):126-31.

11. Perez-Jara J, Walker D, Heslop P, Robinson S. Measuring fear of falling and its effect on quality of life and activity. Rev Clin Gerontol 2010;20(4):277-87.

12. Espy DD, Yang F, Bhatt T, Pai YC. Independent influence of gait speed and step length on stability and fall risk. Gait Posture 2010;32(3):378-82 .

13. Menz HB, Lord SR, Fitzpatrick RC. A structural equation model relating impaired sensorimotor function, fear of falling and gait patterns in older people. Gait Posture 2007;25(2):243-9. 
14. Von Elm E, Altman DG, Egger M, Pocock SJ, Gotzsche PC, Vandenbroucke JP. Strengthening the reporting of observational studies in epidemiology (STROBE) statement: guidelines for reporting observational studies. BMJ 2007;335(7624):806-8.

15. Brucki SMD, Nitrini R, Caramelli P, Bertolucci PHF, Okamoto IH. Sugestões para o uso do mini-exame do estado mental no Brasil. Arq Neuro-Psiquiatr 2003;61(3B):777-8.

16. Almeida MF, Barata RB, Monteiro CV, Silva ZP. Prevalência de doenças crônicas auto-referidas e utilização de serviços de saúde, PNAD/1998, Brasil. Cienc Saude Coletiva 2002;7(4):743-56.

17. Lawton MP, Brody EM. Assessment of older people: self-maintaining and instrumental activities of daily living. Gerontologist 1969;9(3 Part 1):179-86.

18. Katz S, Ford AB, Moskowitz RW, Jackson BA, Jaffe MW. Studies of illness in the aged. The index of ADL: a standardized measure of biological and psychosocial function. JAMA 1963;185:914-9.

19. Camargos FFO, Dias RC, Dias JMD, Freire MTF. Adaptação transcultural e avaliação das propriedades psicométricas da Falls Efficacy Scale - International em idosos brasileiros (FES-I-BRASIL). Rev Bras Fisioter 2010;14(3):237-43.

20. Tinetti ME, Richman D, Powell L. Falls efficacy as a measure of fear of falling. J Gerontol 1990;45(6):239-43.

21. Zijlstra GA, Van Haastregt JC, Van Eijk JT, Van Rossum E, Stalenhoef PA, Kempen GI. Prevalence and correlates of fear of falling, and associated avoidance of activity in the general population of community-living older people. Age Ageing 2007;36(3):304-9.

22. Cesari M. Role of gait speed in the assessment of older patients. JAMA 2011;305(1):93-4.
23. Studenski S, Perera S, Patel K, Rosano C, Faulkner $\mathrm{K}$, Inzitari M, et al. Gait speed and survival in older adults. JAMA 2011;305(1):50-8.

24. Rodrigues IG, Costa GA, Pinto RMC. Qualidade de vida e senso de auto-eficácia para quedas em idosos participantes do Projeto AFRID/UFU. Efdesportes. com [periódico na Internet]. $2009 \mathrm{Fev}$ [ acesso em 26 Jan 2011]; 13(129): [aproximadamente 6 p.]. Disponível em: http://www.efdeportes.com/efd129/ qualidade-de-vida-e-senso-de-auto-eficacia-paraquedas-em-idosos.htm.

25. Custódio EB, Malaquias Júnior J, Voos MC. Relação entre cognição (função executiva e percepção espacial) e equilíbrio de idosos de baixa escolaridade. Fisioter Pesq 2010;17(1)46-51.

26. Soares WJS. Capacidade físico funcional em idosos com e sem história de quedas: projeto fibra [dissertação]. São Paulo: Universidade Cidade de São Paulo; 2009.

27. Deshpande N, Metter EJ, Lauretani F, Bandinelli S, Guralnik J, Ferrucci L. Activity restriction induced by fear of falling and objective and subjective measures of physical function: a prospective cohort study. J Am Geriatr Soc 2008;56(4):615-20.

28. Chamberlin ME, Fulwider BD, Sanders SL, Medeiros JM. Does fear of falling influence spatial and temporal gait parameters in elderly persons beyond changes associated with normal aging? J Gerontol A Biol Sci Med Sci 2005;60(9):1163-7.

29. Rochat S, Büla CJ, Martin E, Seematter-Bagnoud L, Karmaniola A, Aminian K, et al. What is the relationship between fear of falling and gait in wellfunctioning older persons aged 65 to 70 years? Arch Phys Med Rehabil 2010;91(6):879-84.

30. Verghese J, Xue X. Predisability and gait patterns in older adults. Gait Posture 2011;33(1):98-101. 